Revista Destaques Acadêmicos, Lajeado, v. 11, n. 3, 2019. ISSN 2176-3070

DOI: http://dx.doi.org/10.22410/issn.2176-3070.v11i3a2019.2291

http://www.univates.br/revistas

\title{
PERFIL NUTRICIONAL DE IDOSOS EM UM HOSPITAL DO INTERIOR DO RIO GRANDE DO SUL
}

\author{
Rafaela Roman ${ }^{1}$, Bianca Coletti Schauren ${ }^{2}$
}

Resumo: Este estudo teve como objetivo avaliar o perfil nutricional de idosos em um hospital do interior do Rio Grande do Sul. Estudo transversal, de amostragem por conveniência, realizado com indivíduos acima de 60 anos de idade no período de julho a outubro de 2018. Foram coletadas variáveis sobre o perfil demográfico, socioeconômico, antropométrico, avaliação subjetiva global e diagnóstico médico. A análise dos dados foi realizada pelo software Statistical Package for Social Sciences ${ }^{\circledR 22.0 .}$ Para a comparação entre as variáveis categóricas e numéricas foi utilizado o Teste de Associação Exato de Fisher, considerando significativo $p<0,05$. Foram avaliados 116 idosos, com média de idade de 76,6 $\pm 8,8$ anos e 56,9\% do gênero feminino. Quando avaliado o índice de massa corporal, 37,1\% dos idosos encontravam-se eutróficos. Do total, $85,3 \%$ apresentaram risco coronariano aumentado pela circunferência da cintura e pela relação cintura/estatura. A desnutrição pelo índice de massa corporal associou-se significativamente à presença de desnutrição pela circunferência do braço, circunferência da panturrilha, circunferência da cintura, relação cintura estatura e avaliação subjetiva global. A circunferência do braço e panturrilha classifica-se sem risco para redução de massa muscular, respectivamente em 41,4\% e 78,4\%. Conforme avaliação subjetiva global $86,2 \%$ dos idosos encontra-se nutridos. Devido o impacto que as alterações do estado nutricional repercutem sobre a saúde dos idosos hospitalizados destacamos a importância da aplicação de ferramentas para identificação de pacientes com estado nutricional comprometido a fim de promover intervenções precoces que repercutam positivamente sobre o estado nutricional e morbimortalidade.

Palavras-chave: Avaliação nutricional. Estado nutricional. Idoso.

1 Nutricionista, Pós Graduanda em Dietoterapia nos Ciclos da Vida com ênfase em Nutrição Clínica, da Universidade do Vale do Taquari.

2 Nutricionista, Mestre em Ciências da Saúde: Cardiologia IC/FUC-RS, docente do programa de Pós Graduação em Dietoterapia nos Ciclos da Vida com ênfase em Nutrição Clínica, da Universidade do Vale do Taquari. 


\section{INTRODUÇÃO}

O crescimento da população de idosos, em números absolutos e relativos, é um fenômeno mundial e está ocorrendo de forma acelerada. Os números mostram que, atualmente, uma em cada dez pessoas tem 60 anos de idade ou mais e, para 2050, estima-se que a relação será de uma para cinco em todo o mundo, e de uma para três nos países desenvolvidos (IBGE, 2010). No Brasil, estima-se que em 2020 nos aproximaremos de 13 milhões de idosos $(12,4 \%)$ e, se pensarmos em longo prazo, no ano de 2060 mais de um terço da população $(33,7 \%)$ será constituído por pessoas com 60 anos ou mais (IBGE, 2013).

Concomitantes ao processo de envelhecimento ocorrem manifestações clínicas e surgimento de doenças crônicas degenerativas, que repercutem sobre a qualidade de vida e estado nutricional dos idosos. Conforme estudo realizado em 131 idosos internados, com faixa etária de 60 a 95 anos, observou-se que a grande maioria dos pacientes, de ambos os gêneros, tem como principal motivo de internação o câncer $(38,17 \%)$, seguido de doenças cardiovasculares $(27,48 \%)$, apresentando a hipertensão arterial sistêmica como doença associada (ZANCHIM, LIBERALI, COUTINHO, 2013).

Outro estudo realizado com idosos internados sendo 116 indivíduos com idade média de 78 anos, sendo $84,3 \%$ com mais de 70 anos e $30,2 \%$ na faixa de muito idosos, identificou-se que entre as principais causas de internação foram as doenças cardíacas (19\%), seguidas das doenças urológicas $(14,7 \%)$ e pulmonares $(13,8 \%)$, sendo que $58,6 \%$ desses pacientes eram hipertensos e $25 \%$ tinham diabetes (SILVA, MANNARINO, MOREIRA, 2012).

O motivo e tempo de internação podem afetar diretamente o estado nutricional, sendo que no paciente hospitalizado, a desnutrição pode se instalar rapidamente devido ao estado de hipercatabolismo que acompanha as enfermidades, traumatismos e infecções em resposta ao estresse metabólico que ocorre nestas condições, principalmente quando a ingestão nutricional é insuficiente (PORTAL DA SAÚDE, 2019).

Em estudo com 131 idosos hospitalizados demostrou as classificações do estado nutricional, sendo 50,39\% eutróficos, 36,64\% magreza e 12,97\% em excesso de peso (ZANCHIM, LIBERALI, COUTINHO, 2013). Já o estudo de BRAINS, constatou uma frequência de desnutrição, através da análise exclusiva da ASG, de $24 \%$. Destes, $18,3 \%$ dos pacientes encontravam-se moderadamente desnutridos ou com suspeita de desnutrição e 5,7\%, gravemente desnutridos (MEALE et.al 2013).

Portanto, no ambiente hospitalar, a identificação dos pacientes com comprometimento do estado nutricional é fundamental para o diagnóstico e aplicação precoce de intervenções nutricionais capazes de promover reabilitação e recuperação do paciente desnutrido (HENRICHSEN, MORAES, SCHAUREN, 2017), pois o estado nutricional interfere diretamente na evolução 
clínica do paciente hospitalizado gerando maior tempo de internação e custos hospitalares (OLIVEIRA, 2015).

Diante do exposto, a avaliação do estado nutricional do paciente hospitalizado é fundamental na medida em que se reconhece a alta prevalência de risco nutricional (CRESTANI et.al 2011). Neste sentido, o objetivo do presente estudo foi avaliar o perfil nutricional de idosos hospitalizados em um município do interior do Rio Grande do Sul.

\section{MÉTODOS}

Trata-se de um estudo do tipo quantitativo, descritivo e analítico, com delineamento transversal realizado em um município do interior do Rio Grande do Sul através da análise de dados primários e secundários. A amostragem foi concentrada em uma instituição hospitalar que possui 83 leitos, com média de 213 internações mensais em 2017, destas 88 internações correspondem a idosos de ambos os gêneros.

Os idosos internados foram convidados a participar da pesquisa por meio das visitas realizadas pela Nutricionista. Fizeram parte da amostra 116 idosos, atendendo aos critérios de inclusão: indivíduos acima de 60 anos de idade, de ambos os gêneros, internados entre os meses de julho a outubro de 2018, período do ano no qual se identificam maiores índices de internação. Foram excluídos aqueles que não puderam ou quiseram participar de alguma etapa da pesquisa e indivíduos acamados, cadeirantes ou impossibilitados de realizar a avaliação antropométrica.

Foi aplicado um questionário estruturado com dados referentes às variáveis de perfil demográfico, perfil socioeconômico, Índice de Massa Corporal (IMC), Circunferência do Braço (CB), Circunferência da Panturrilha (CP), Circunferência da Cintura (CC), Relação cintura estatura (Rcest) e Avaliação Nutricional Subjetiva Global (ANSG). O diagnóstico médico, por tratar-se de um dado secundário, foi coletado no prontuário eletrônico, conforme dados da prescrição médica e classificado conforme as especialidades médicas.

A massa corporal e estatura foram aferidas no ato da realização das entrevistas, da seguinte forma: massa corporal, obtida utilizando-se balança digital marca Plenna ${ }^{\circledR}$, com capacidade de $150 \mathrm{~kg}$ e precisão de $100 \mathrm{~g}$ (Plenna Especialidades Ltda, São Paulo, Brasil), conforme o procedimento padrão da Word Health Organization (1995). A medida da estatura foi obtida utilizando-se estadiômetro portátil da marca SECA $®$, modelo 208, com capacidade de 200 cm e precisão de $0,1 \mathrm{~cm}$ (Seca, Hamburgo, Alemanha), conforme procedimento padrão International Standards for Anthropometric Assessment (2001).

As aferições da CB, CP e CC foram obtidas utilizando uma trena antropométrica com capacidade de $2 \mathrm{~m}$ (Wiso®, Santa Catariana, Brasil) conforme procedimento padrão International Standards for Anthropometric Assessment (2001). 
Posteriormente a coleta, foi realizada a avaliação do estado nutricional, através do IMC (OPAS, 2002). Foi avaliada a classificação do estado nutricional conforme a adequação da Circunferência do Braço (FRISANCHO, 1981). A classificação da CC foi obtida conforme parâmetros preconizados pela WHO (2000) e classificação da Rcest obtida pelos parâmetros de (PITANGA 2011). A classificação da $\mathrm{CP}$ foi considerada adequada a $\mathrm{CP} \geq 31 \mathrm{~cm}$ para homens ou mulheres (ASHWELL, GIBSON, 2009). E por fim, a avaliação do estado nutricional foi realizada através da Avaliação Nutricional Subjetiva Global (DIAS et al., 2011).

A obtenção das informações ocorreu somente após aprovação do Comitê de Ética em Pesquisa da Universidade do Vale do Taquari, sob o registro número 2.674.311. Este estudo seguiu as recomendações da Resolução 466/2012 do Conselho Nacional de Saúde.

A análise dos dados foi realizada pelo software Statistical Package for the Social Sciences (SPSS®) versão 22.0 através de estatísticas descritivas e para avaliação da associação das variáveis o teste estatístico de Associação Exato de Fisher. Os resultados foram considerados significativos a um nível de significância máximo de $5 \%(p \leq 0,05)$.

\section{RESULTADOS}

Foram avaliados 116 idosos, com média de idade de $76,6 \pm 8,8$ anos, sendo que a maioria $(39,7 \%)$ possuía entre 70 a 80 anos, 56,9\% do gênero feminino, $56,0 \%$ eram casadas ou moravam com um companheiro. No que se refere à escolaridade $68,1 \%$ possuíam 0 a 4 anos de estudo, $98,3 \%$ eram aposentados e $87,1 \%$ informou ter renda de até 1 salário mínimo (TABELA 1).

Tabela 1. Caracterização da amostra, segundo dados demográficos e socioeconômicos de idosos de um município do interior do Rio Grande do Sul, 2018

\begin{tabular}{llcc}
\hline Variáveis & & $\mathrm{n}$ & $(\%)$ \\
\hline \multirow{3}{*}{ Idade } & 70 anos & 29 & $(25,0)$ \\
& $70-80$ anos & 46 & $(39,7)$ \\
& $\geq 80$ anos & 41 & $(35,3)$ \\
\hline \multirow{2}{*}{ Gênero } & Feminino & 66 & $(56,9)$ \\
& Masculino & 50 & $(43,1)$ \\
\hline \multirow{3}{*}{ Estado civil } & Solteiro & 4 & $(3,4)$ \\
& Casado /Com companheiro & 65 & $(56,0)$ \\
& Separado/divorciado/desquitado & 4 & $(3,4)$ \\
& Viúvo & 43 & $(37,1)$ \\
\hline
\end{tabular}




\begin{tabular}{|c|c|c|c|}
\hline Variáveis & & $\mathrm{n}$ & $(\%)$ \\
\hline \multirow{4}{*}{ Escolaridade } & $0-4$ anos & 79 & $(68,1)$ \\
\hline & $5-8$ anos & 26 & $(22,4)$ \\
\hline & $9-12$ anos & 5 & $(4,3)$ \\
\hline & $\geq 13$ anos & 6 & $(5,2)$ \\
\hline \multirow{3}{*}{$\begin{array}{l}\text { Ocupação } \\
\text { profissional }\end{array}$} & Funcionário público & 1 & $(0,9)$ \\
\hline & Aposentado (a) & 114 & $(98,3)$ \\
\hline & Não trabalho & 1 & $(0,9)$ \\
\hline \multirow{3}{*}{ Renda } & Até 1 s.m & 101 & $(87,1)$ \\
\hline & De 1,1 até 2 s.m & 14 & $(12,1)$ \\
\hline & Nenhuma renda & 1 & $(0,9)$ \\
\hline
\end{tabular}

Legenda: $\mathrm{n}$ = número; $\%$ = porcentagem; s.m = salário mínimo.

Dentre os diagnósticos médicos mais prevalentes encontrados através das especialidades médicas, podem-se observar as doenças pulmonares $(41,4 \%)$, doenças gastrointestinais $(8,6 \%)$, doenças cardiovasculares $(7,8 \%)$ e doenças ortopédicas $(5,2 \%)$. Quando avaliados os pacientes de acordo com a especialidade médica associados a ASG, a prevalência de desnutrição foi maior naqueles acometidos por doenças pulmonares (53,3\%).

Com relação ao estado nutricional, verificou-se que o IMC médio foi $27,0 \pm 6,3 \mathrm{Kg} / \mathrm{m}^{2}$, sendo que a maioria se encontrava eutrófico $(37,1 \%)$. No entanto, quando avaliado o restante dos participantes, $27,6 \%$ dos idosos avaliados já apresentavam desnutrição e 35,4\% foram identificados com excesso de peso (sobrepeso e obesidade). Em relação a CB, embora 41,4\% estavam eutróficos, observa-se que $21,6 \%$ dos pacientes foram diagnósticas com obesidade e 12,1\% com desnutrição leve. Já a CP, 78,4\% dos idosos foram classificados como adequado em relação a quantidade de massa muscular e, tanto a CC e Rcest, 85,3\% apresentaram risco coronariano aumentado. No que se refere a classificação da ASG, apesar de $86,2 \%$ terem sido identificados como bem nutridos, $12,9 \%$ dos pacientes apresentaram desnutrição moderada ou grave (TABELA 2).

Tabela 2. Características Nutricionais de idosos de um município do interior do Rio Grande do Sul, 2018

\begin{tabular}{llcc}
\hline Variáveis & & $\mathrm{n}$ & $(\%)$ \\
\hline \multirow{3}{*}{ IMC } & Desnutrição & 32 & $(27,6)$ \\
& Eutrofia & 43 & $(37,1)$ \\
& Sobrepeso & 14 & $(12,1)$ \\
& Obesidade & 27 & $(23,3)$ \\
\hline
\end{tabular}




\begin{tabular}{llcc}
\hline Variáveis & & $\mathrm{n}$ & $(\%)$ \\
\hline \multirow{4}{*}{$\mathrm{CB}$} & Desnutrição grave & 4 & $(3,4)$ \\
& Desnutrição moderada & 12 & $(10,3)$ \\
& Desnutrição leve & 14 & $(12,1)$ \\
& Eutrofia & 48 & $(41,4)$ \\
& Sobrepeso & 13 & $(11,2)$ \\
$\mathrm{CP}$ & Obesidade & 25 & $(21,6)$ \\
\hline \multirow{2}{*}{$\mathrm{CC}$} & Adequado & 91 & $(78,4)$ \\
& Diminuído & 25 & $(21,6)$ \\
\hline \multirow{2}{*}{ Rcest } & Risco coronariano & 99 & $(85,3)$ \\
& Sem risco coronariano & 17 & $(14,7)$ \\
\hline \multirow{2}{*}{ ASG } & Risco coronariano & 99 & $(85,3)$ \\
& Sem risco coronariano & 17 & $(14,7)$ \\
\hline
\end{tabular}

Legenda: $\mathrm{n}=$ número; \% = porcentagem; $\mathrm{IMC}=$ índice de massa corporal; $\mathrm{CB}=$ circunferência do braço; $\mathrm{CP}$ = circunferência da panturrilha; $\mathrm{CC}=$ circunferência da cintura; Rcest = relação cintura estatura; ASG = Avaliação Subjetiva Global.

Conforme descrito na Tabela 3, evidenciou-se associação significativa entre o IMC com as variáveis CB, CP, CC, Rcest e a ASG. Pacientes com desnutrição segundo o IMC estiveram significativamente associadas ao diagnóstico de desnutrição moderada e grave segundo a $C B(p<0,001)$ enquanto os pacientes com sobrepeso e obesidade ao diagnóstico de sobrepeso e obesidade conforme a CB ( $\mathrm{p}<0,001)$. Idosos diagnosticados com diminuição da massa muscular pela $\mathrm{CP}$ estiveram associados ao diagnóstico de desnutrição segundo o IMC e a adequação da CP ao IMC de eutrofia, sobrepeso e obesidade $(\mathrm{p}<0,001)$.

Tabela 3. Associações de dados demográficos, socioeconômicos e características nutricionais com Índice de Massa corporal de idosos de um município do interior do Rio Grande do Sul, 2018

\begin{tabular}{|c|c|c|c|c|c|c|c|c|c|c|}
\hline \multirow{2}{*}{ Variável } & \multirow{2}{*}{ Resposta } & \multicolumn{2}{|c|}{ Desnutrição } & \multicolumn{2}{|c|}{ Eutrofia } & \multicolumn{2}{|c|}{ Sobrepeso } & \multicolumn{2}{|c|}{ Obesidade } & \multirow[b]{2}{*}{$\mathrm{p}$} \\
\hline & & $\mathrm{n}$ & $(\%)$ & $\mathrm{n}$ & $(\%)$ & $\mathrm{n}$ & $(\%)$ & $\mathrm{n}$ & $(\%)$ & \\
\hline \multirow{3}{*}{ Idade } & 70 anos & 6 & $(18,8)$ & 10 & $(23,3)$ & 7 & $(50,0)$ & 6 & $(22,2)$ & 0,106 \\
\hline & $70-80$ anos & 15 & $(46,9)$ & 16 & $(37,2)$ & 1 & $(7,1)$ & 14 & $(51,9)$ & \\
\hline & $\geq 80$ anos & 11 & $(34,4)$ & 17 & $(39,5)$ & 6 & $(42,9)$ & 7 & $(25,9)$ & \\
\hline \multirow{2}{*}{ Gênero } & Feminino & 17 & $(53,1)$ & 22 & $(51,2)$ & 10 & $(71,4)$ & 17 & $(63,0)$ & 0,507 \\
\hline & Masculino & 15 & $(46,9)$ & 21 & $(48,8)$ & 4 & $(28,6)$ & 10 & $(37,0)$ & \\
\hline
\end{tabular}




\begin{tabular}{|c|c|c|c|c|c|c|c|c|c|c|}
\hline \multirow{2}{*}{ Variável } & \multirow{2}{*}{ Resposta } & \multicolumn{2}{|c|}{ Desnutrição } & \multicolumn{2}{|c|}{ Eutrofia } & \multicolumn{2}{|c|}{ Sobrepeso } & \multicolumn{2}{|c|}{ Obesidade } & \multirow{2}{*}{$\mathrm{p}$} \\
\hline & & $\mathrm{n}$ & $(\%)$ & $\mathrm{n}$ & $(\%)$ & $\mathrm{n}$ & $(\%)$ & $\mathrm{n}$ & $(\%)$ & \\
\hline \multirow{4}{*}{ Estado civil } & Solteiro & 3 & $(9,4)$ & - & - & - & - & 1 & $(3,7)$ & 0,220 \\
\hline & $\begin{array}{l}\text { Casado / com } \\
\text { companheiro }\end{array}$ & 14 & $(43,8)$ & 29 & $(67,4)$ & 8 & $(57,1)$ & 14 & $(51,9)$ & \\
\hline & $\begin{array}{l}\text { Separado/ } \\
\text { divorciado }\end{array}$ & 3 & $(9,4)$ & 1 & $(2,3)$ & - & - & - & - & \\
\hline & Viúvo & 12 & $(37,5)$ & 13 & $(30,2)$ & 6 & $(42,9)$ & 12 & $(44,4)$ & \\
\hline \multirow{4}{*}{ Escolaridade } & $0-4$ anos & 24 & $(75,0)$ & 26 & $(60,5)$ & 10 & $(71,4)$ & 19 & $(70,4)$ & 0,475 \\
\hline & $5-8$ anos & 5 & $(15,6)$ & 13 & $(30,2)$ & 3 & $(21,4)$ & 5 & $(18,5)$ & \\
\hline & $9-12$ anos & 2 & $(6,3)$ & - & - & 1 & $(7,1)$ & 2 & $(7,4)$ & \\
\hline & $\geq 13$ anos & 1 & $(3,1)$ & 4 & $(9,3)$ & - & - & 1 & $(3,7)$ & \\
\hline \multirow{3}{*}{ Renda } & Até 1 s.m & 29 & $(90,6)$ & 37 & $(86,0)$ & 12 & $(85,7)$ & 23 & $(85,2)$ & 0,533 \\
\hline & De 1,1 até 2 s.m & 3 & $(9,4)$ & 6 & $(14,0)$ & 1 & $(7,1)$ & 4 & $(14,8)$ & \\
\hline & Nenhuma renda & - & - & - & - & 1 & $(7,1)$ & - & - & \\
\hline \multirow{6}{*}{$\mathrm{CB}$} & Desnutrição grave & 3 & $(9,4)$ & - & - & - & - & 1 & $(3,7)$ & $<0,001$ \\
\hline & $\begin{array}{l}\text { Desnutrição } \\
\text { moderada }\end{array}$ & 9 & $(28,1)$ & 2 & $(4,7)$ & 1 & $(7,1)$ & - & - & \\
\hline & Desnutrição leve & 6 & $(18,8)$ & 6 & $(14,0)$ & - & - & 2 & $(7,4)$ & \\
\hline & Eutrofia & 9 & $(28,1)$ & 25 & $(58,1)$ & 7 & $(50,0)$ & 7 & $(25,9)$ & \\
\hline & Sobrepeso & 1 & $(3,1)$ & 4 & $(9,3)$ & 4 & $(28,6)$ & 4 & $(14,8)$ & \\
\hline & Obesidade & 4 & $(12,5)$ & 6 & $(14,0)$ & 2 & $(14,3)$ & 13 & $(48,1)$ & \\
\hline \multirow{2}{*}{$\mathrm{CP}$} & Adequado & 5 & $(46,9)$ & 38 & $(88,4)$ & 13 & $(92,9)$ & 25 & $(92,6)$ & $<0,001$ \\
\hline & Diminuído & 17 & $(53,1)$ & 5 & $(11,6)$ & 1 & $(7,1)$ & 2 & $(7,4)$ & \\
\hline \multirow[b]{2}{*}{$\mathrm{CC}$} & Risco coronariano & 16 & $(50,0)$ & 42 & $(97,7)$ & 14 & $(100)$ & 27 & $(100,0)$ & $<0,001$ \\
\hline & $\begin{array}{l}\text { Sem risco } \\
\text { coronariano }\end{array}$ & 16 & $(50,0)$ & 1 & $(2,3)$ & - & - & - & - & \\
\hline \multirow[b]{2}{*}{ Rcest } & Risco coronariano & 18 & $(56,3)$ & 41 & $(95,3)$ & 14 & (100) & 26 & $(96,3)$ & $<0,001$ \\
\hline & $\begin{array}{l}\text { Sem risco } \\
\text { coronariano }\end{array}$ & 14 & $(43,8)$ & 2 & $(4,7)$ & - & - & 1 & $(3,7)$ & \\
\hline \multirow{3}{*}{ ASG } & Nutrido & 16 & $(50,0)$ & 43 & $(00,0)$ & 14 & (100) & 27 & $(100,0)$ & $<0,001$ \\
\hline & $\begin{array}{l}\text { Moderadamente } \\
\text { desnutrido ou } \\
\text { suspeita de } \\
\text { desnutrição }\end{array}$ & 15 & $(46,9)$ & - & - & - & - & - & - & - \\
\hline & $\begin{array}{l}\text { Gravemente } \\
\text { desnutrido }\end{array}$ & 1 & $(3,1)$ & - & - & - & - & - & - & - \\
\hline
\end{tabular}

Legenda: $\mathrm{n}$ = número; \% = porcentagem; s.m = salário mínimo; Rcest = relação cintura estatura; $\mathrm{CB}=$ circunferência braço; $\mathrm{CP}$ = circunferência panturrilha; $\mathrm{CC}$ = circunferência cintura; $\mathrm{ASG}=$ Avaliação Subjetiva Global.

Quanto a CC e Rcest, valores elevados preditivos de risco coronariano aumentado foram associados aos idosos com IMC de eutrofia, sobrepeso e obesidade $(p<0,001)$ e a CC e Rcest sem risco aos idosos com desnutrição $(\mathrm{p}<0,001)$.

$\mathrm{Na}$ Tabela 4, conforme a análise comparativa entre parâmetros antropométricos e as classificações da ASG, observou-se que pacientes moderadamente desnutridos ou com suspeita de desnutrição estiveram 
associados a desnutrição leve, grave e moderada conforme a $C B(p<0,001)$ e diminuição da massa muscular segundo a $\mathrm{CP}(\mathrm{p}<0,001)$. Os idosos bem nutridos apresentaram valores normais de CC $(\mathrm{p}<0,001)$ e Rcest $(\mathrm{p}<0,001)$.

Tabela 4 - Associações de idade, gênero e características nutricionais com Avaliação Nutricional Subjetiva Global de idosos de um município do interior do Rio Grande do Sul, 2018

\begin{tabular}{|c|c|c|c|c|c|c|c|c|}
\hline \multirow[t]{2}{*}{ Variáveis } & \multirow[t]{2}{*}{ Resposta } & \multicolumn{2}{|c|}{ Nutrido } & \multicolumn{2}{|c|}{$\begin{array}{c}\text { Moderadamente } \\
\text { desnutrido ou } \\
\text { suspeita de } \\
\text { desnutrição }\end{array}$} & \multicolumn{2}{|c|}{$\begin{array}{l}\text { Gravemente } \\
\text { desnutrido }\end{array}$} & \multirow[t]{2}{*}{$\mathrm{p}$} \\
\hline & & $\mathrm{n}$ & $(\%)$ & $\mathrm{n}$ & $(\%)$ & $\mathrm{n}$ & $(\%)$ & \\
\hline \multirow[t]{3}{*}{ Idade } & $<70$ anos & 28 & $(28)$ & 1 & $(6,7)$ & - & - & 0,144 \\
\hline & $70-80$ anos & 36 & (36) & 9 & $(60,0)$ & 1 & $(100,0)$ & \\
\hline & $>80$ anos & 36 & (36) & 5 & $(33,3)$ & - & - & \\
\hline \multirow[t]{4}{*}{ Gênero } & Feminino & 57 & (57) & 9 & $(60,0)$ & - & - & 0,760 \\
\hline & Masculino & 43 & (43) & 6 & $(40,0)$ & 1 & $(100,0)$ & \\
\hline & Desnutrição grave & 1 & $(1,0)$ & 2 & $(13,3)$ & 1 & $(100,0)$ & $<0,001$ \\
\hline & Desnutrição moderada & 5 & $(5,0)$ & 7 & $(46,7)$ & - & - & \\
\hline \multirow[t]{4}{*}{$\mathrm{CB}$} & Desnutrição leve & 10 & (10) & 4 & $(26,7)$ & - & - & \\
\hline & Eutrofia & 47 & $(47)$ & 1 & $(6,7)$ & - & - & \\
\hline & Sobrepeso & 12 & (12) & 1 & $(6,7)$ & - & - & \\
\hline & Obesidade & 25 & (25) & - & - & - & - & \\
\hline \multirow{2}{*}{$\mathrm{CP}$} & Adequado & 87 & (87) & 4 & $(26,7)$ & - & - & $<0,001$ \\
\hline & Diminuído & 13 & (13) & 11 & $(73,3)$ & 1 & $(100,0)$ & \\
\hline \multirow{2}{*}{$\mathrm{CC}$} & Risco coronariano & 93 & (93) & 6 & $(40,0)$ & - & - & $<0,001$ \\
\hline & Sem risco coronariano & 7 & $(7,0)$ & 9 & $(60,0)$ & 1 & $(100,0)$ & \\
\hline \multirow{2}{*}{ Rcest } & Risco coronariano & 91 & (91) & 8 & $(53,3)$ & - & - & $<0,001$ \\
\hline & Sem risco coronariano & 9 & $(9,0)$ & 7 & $(46,7)$ & 1 & $(100,0)$ & \\
\hline
\end{tabular}

Legenda: $\mathrm{n}=$ número; $\%$ = porcentagem; $\mathrm{CB}=$ circunferência do braço; $\mathrm{CP}=$ circunferência da panturrilha; $\mathrm{CC}$ = circunferência da cintura; Rcest = relação cintura estatura.

\section{DISCUSSÃO}

No presente estudo, evidenciou-se que a maioria dos participantes eram mulheres com idade entre 70 e 80 anos. Estes achados corroboram com os dados da Pesquisa de Orçamentos Familiares (POF 2008/2009), que buscou diagnosticar o estado nutricional da população brasileira idosa e encontrou $55,6 \%$ da amostra composta por mulheres. No entanto, quanto à média etária, registrou 56\% de idosas mais jovens, entre 60 a 69 anos (PEREIRA, SPYRIDES, ANDRADE, 2016). Já Zanchin, Liberali e Coutinho, em estudo realizado com 
131 idosos hospitalizados em um hospital geral, verificou que a maioria dos participantes eram homens $(55,73 \%)$, mas com idade média semelhante aos achados do presente estudo, 71,1 $\pm 7,40$ anos para homens e 70,5 $\pm 6,69$ anos para as mulheres, respectivamente (ZANCHIN, LIBERALI, COUTINHO, 2013). Estes achados estão ao encontro dos últimos estudos sobre o perfil da população brasileira, com aumento dos anos de vida e da proporção de mulheres, as quais apresentam maior expectativa de vida aos 60 anos $(23,9 \%)$ se comparado com os homens 20,3\% (IBGE, 2018).

Quanto ao diagnóstico médico encontrado, em consonância com o estudo de Tonini, que objetivou avaliar o estado nutricional de idosos hospitalizados, registrou achado semelhante ao presente estudo, sendo o diagnóstico médico mais evidente foi relacionado às doenças pulmonares $(41,1 \%)$ (TONINI, 2018). Se relacionado com outro estudo a maioria dos pacientes era acometida por desordens gastrointestinais $(n=145 ; 24,8 \%)$, neoplasias $(n=103 ; 17,6 \%)$, patologias cardíacas $(n=64 ; 10,9 \%)$, neurológicas $(n$ $=52 ; 8,9 \%)$, vasculares $(n=50 ; 8,5 \%)$ e por problemas renais $(n=49 ; 8,4 \%)$, não sendo diagnosticados pacientes com doenças pulmonares conforme achado do presente estudo (HENRICHSEN, MORAES, SCHAUREN, 2017). Quando avaliado as intercorrências na população idosa, as complicações no sistema cardíaco, respiratório e nervoso são registradas como as principais complicações relacionadas ao processo de envelhecimento (FECHINE, TROMPIERI, 2012).

Em relação ao estado nutricional avaliado pelo IMC, pode-se encontrar contrariedade do presente estudo com os achados encontrados nos 212 idosos hospitalizados avaliados, no qual 49,6\% estavam desnutridos, 32,5\% eutróficos e 17,9\% obesos, sem diferença entre homens e mulheres (PALA et.al 2011). $\mathrm{O}$ mesmo foi encontrado em estudo realizado com 116 idosos internados, com média de idade de 78 anos, mostrou que $26,7 \%$ eram classificados como desnutridos pelo IMC já no momento da admissão hospitalar (SILVA, MANNARINO, MOREIRA, 2012). Sendo assim pode-se evidenciar que a desnutrição não é a única forma de classificação do estado nutricional dos idosos hospitalizados, e sim, deve-se também atentar para prevalências de excesso de peso e obesidade, associado a outros métodos de avaliação do estado nutricional nesta população (TRENTIN, BERNARDI, SIVIERO, 2016).

Conforme o resultado obtido pela $\mathrm{CB}$ no presente estudo pode identificar contrariedade conforme estudo realizado em apenas 69 idosos de ambos os gêneros, apontando que mais que a metade $(54,1 \%)$ dos avaliados apresentou desnutrição pela CB (CASTRO DA PAZ, FAZZIO, SANTOS, 2012). Em comparação com estudo realizado em 445 pacientes internados, destes $38,9 \%$ caracterizados por idosos, a prevalência total de desnutrição conforme o critério da CB foi de $41,4 \%$, percentual maior de desnutrição se comparado com os critérios de IMC (15,5\%) e ASG (39,8\%) (MARCADENTI, 2011). Considerando que a avaliação antropométrica isolada de apenas um indicador não deve ser usada como método para detectar desnutrição hospitalar, mas 
um conjunto de indicadores que possibilitem uma análise mais detalhada do estado nutricional devido a maior prevalência de desnutrição que ocorre entre os idosos acima dos 70 anos de idade, com diminuição significativa de outros parâmetros antropométricos com o decorrer do aumento da idade (PALA et.al 2011).

Os idosos hospitalizados no presente estudo, em sua maioria, apresentaram adequada classificação conforme critério antropométrico da $\mathrm{CP}$, achado semelhante ao estudo realizado com 70 idosos oncológicos internados em um hospital de alta complexidade, sendo $65,7 \%$ diagnosticaram maior proporção de eutrofia (PELISSARO et.al 2016). No entanto deve-se considerar a CP como uma medida para identificação precoce de diminuição da massa muscular em avaliações de rotina de idosos na atenção primária (PAGOTO et.al 2018).

Dentre os demais critérios antropométricos avaliados no presente estudo, a CC e Rcest encontraram-se risco coronariano aumentado nos idosos. Conforme estudo realizado em 134 idosos, sendo maioria do gênero feminino, apresentaram achados semelhantes de $83,6 \%$ de circunferência abdominal aumentada, entre as faixas etárias de 70-80 anos (DINIZ, TAVARES, 2013). Sendo assim devemos atentar que valores acima dos parâmetros preconizados para CC e Rcest são preditores de risco para doença cardiovascular, aumento da glicemia e da pressão arterial correlacionando com a baixa adesão a práticas de estilo de vida saudáveis que podem contribuir para a prevenção do agravamento desses fatores de risco (AUDI et.al 2016).

De acordo com a avaliação subjetiva global, evidenciou-se que aproximadamente um sexto da amostra apresentou desnutrição moderada ou grave. A presença de desnutrição em pacientes internados também foi observada em estudo no qual a desnutrição moderada foi evidenciada em $24,6 \%$ dos pacientes, enquanto que $8,4 \%$ dos pacientes foram classificados como desnutridos graves (HENRICHSEN, MORAES, SCHAUREN, 2017). Já em estudo realizado com 39 pacientes adultos e idosos de um hospital público, revelam uma preocupante realidade do grupo amostral investigado, sendo observado que todos os avaliados apresentaram grau de desnutrição grave (AZEVEDO, OLIVEIRA, SOARES, 2016). Os resultados do presente estudo reforçam os achados da literatura, evidenciando que a presença da desnutrição é uma realidade no ambiente hospitalar. A avaliação do risco nutricional através de ferramentas de triagem nutricional é indispensável em indivíduos hospitalizados, para que estes recebam a atenção nutricional apropriada (PEREIRA et.al, 2014).

\section{CONCLUSÃO}

A partir dos resultados do presente estudo podemos evidenciar que a prevalência de idosos hospitalizados que apresentam alterações do estado 
nutricional ainda é preocupante, conforme as associações encontradas no estudo, evidenciando a relação entre a presença de alterações do estado nutricional pelos diferentes métodos: IMC, CB, CP, CC, Rcest e a ASG. Neste sentido, destaca-se a importância da aplicação de ferramentas como ASG para identificação de pacientes com estado nutricional comprometido a fim de promover intervenções precoces que repercutam positivamente sobre o estado nutricional e morbimortalidade dos pacientes.

Além disso, a atuação de uma equipe multiprofissional, desde o momento da internação até a alta hospitalar, que melhor atenda às necessidades do paciente com vistas à promoção e recuperação da saúde, pode impactar no prognóstico clínico, diminuir o tempo de internação e os custos hospitalares.

\section{REFERÊNCIAS}

ASHWELL, M.; GIBSON, S.; Waist to Height Ratio Is a Simple and Effective Obesity Screening Tool for Cardiovascular Risk Factors: Analysis of Data from the British National Diet and Nutrition Survey of Adults Aged 19-64 Years. Obes Facts. 2009; 2: 97-103

AUDI, CAF, SANTIAGO, SM, ANDRADE, MGGA, FRANCISCO, PMSB, Fatores de risco para doenças cardiovasculares em servidores de instituição prisional: estudo transversal. Revista de epidemiologia Serviço da Saúde, 2016.

AZEVEDO, A.S.; OLIVEIRA, D.C.; SOARES, K.D.S.; Perfil Nutricional de adultos e idosos hospitalizados. Revista Saúde e Pesquisa, 2016.

BRASIL, PORTAL DA SAÚDE, Atenção Especializada e Hospitalar, 2019.

Disponível em: http://portalms.saude.gov.br/atencao-especializada-e-hospitalar/ especialidades/desnutricao

CASTRO DA PAZ, R.; FAZZIO, D.M.G.; SANTOS, A.L.B.; Avaliação nutricional de idosos hospitalizados. Revisa, 2012.

CRESTANI, $\mathrm{N}$ et al. Perfil nutricional de pacientes adultos e idosos admitidos em um hospital universitário. Rev. Ciência \& Saúde, Porto Alegre, v. 4, n. 2, p. 45-49, 2011.

DINIZ, M.A.; SANTOS, D.M.; Fatores de risco para doenças cardiovasculares em idosos de um município do interior de Minas Gerais, Texto contexto Enfer, 2013.

FECHINE, B.R.A.; TROMPIERI, N.; O processo de envelhecimento: as principais alterações que acontecem com o passar dos anos. Revista Científica Internacional Interscienceplace, Ceará, 2012.

HENRICHSEN, J.; MORAES, S.F.; SCHAUREN, B.C.; Prevalência de desnutrição em pacientes adultos que ingressam na emergência de um hospital público. Rev. Nutr. Clí. Diet. Hosp. 2017. 
INSTITUTO BRASILEIRO DE GEOGRAFIA E ESTATÍSTICA. Estatísticas de Gênero - Indicadores sociais das mulheres no Brasil, 2018, Disponível em: https:/ /www.ibge. gov.br/estatisticas/multidominio/genero/20163-estatisticas-de-genero-indicadoressociais-das-mulheres-no-brasil.html $?=\& \mathrm{t}=\mathrm{o}-\mathrm{que}-\mathrm{e}$

INSTITUTO BRASILEIRO DE GEOGRAFIA E ESTATÍSTICA. Pesquisa Nacional por Amostra de Domicílios - um panorama da Saúde no Brasil: acesso e utilização dos serviços, condições de saúde e fatores de risco e proteção à saúde (PNAD 2008). Rio de Janeiro: IBGE, 2010.

INSTITUTO BRASILEIRO DE GEOGRAFIA E ESTATÍSTICA. Síntese de indicadores sociais: uma análise das condições de vida da população brasileira [Internet]. Rio de Janeiro: Instituto Brasileiro de Geografia e Estatística; 2013. (Estudos e Pesquisas Informação Demográfica e Socioeconômica, 32). Disponível em: http:/ / biblioteca. ibge.gov.br/visualizacao/livros/liv66777.pdf.

MARCANDENT, A.; VENCATTO, C.; BOUCINHA, M.E.; LEUCH, M.P.; RABELLO, R.; LONDERO, L.G.; RIBEIRO, A.S.; Desnutrição, tempo de internação e mortalidade em um hospital geral do sul do Brasil. Revista Ciência e Saúde, 2011.

MEALE, M.M.S.; GOUVEIA, M.A.P.; FRANÇA, J.I.D.; DAMIÃO, A.O.M.C.; Perfil Nutricional de pacientes internados no Brasil: análise de 19.222 pacientes (estudo BRAINS). Rev. Bras. Nutr. Clin. 2013; 28 (4): 255-63.

OLIVEIRA, T.R.; FORTES, R.C.; Prevalência de desnutrição em pacientes cirúrgicos em terapia nutricional e sua relação com os parâmetros objetivos e subjetivos da avaliação nutricional. Com. Ciências Saúde. 2015; 26(3/4): 115-126.

PAGOTOOL, V, SANTOS, KF, MALAQUIASL, SG, BACHIONL, MM, SILVEIRA, EA, Circunferência da panturrilha: validação clínica para avaliação de massa muscular em idosos. Revista Brasileira de Enfermagem, 2018.

PALA, D.; FREITAS, S.L.; BASTOS ARAÚJO, A.Q.; SILVA, W.M.; AGUIAR NEMER, A.S.; Perfil Nutricional de Idosos hospitalizados. Rev. Bras. Nutr. Clin. 2011; 26 (4): 227-33.

PELISSARO, E.; DAMO, C.C.; ALVES, A.L.S.; CALCING, A.; KUMPEL, D.A.; Avaliação do estado nutricional em pacientes idosos oncológicos internados em um hospital de alta complexidade do norte do Rio Grande do Sul. Revista Scientia Medica, 2016.

PEREIRA, I.F.S.; SPYRIDES, M.H.C.; ANDRADE, L.M.B.; Estado nutricional de idosos do Brasil: uma abordagem multinível. Caderno Saúde Pública, Rio de Janeiro, 2016.

PEREIRA, JD, PALUDO, J, LEMOS JRN, MOREIRA, TR, Variação da prevalência de risco nutricional em indivíduos hospitalizados conforme cinco protocolos de triagem nutricional. Scientia Medica, 2014. 
PITANGA, F, J, G.; Antropometria na avaliação da obesidade abdominal e risco coronariano. Universidade Federal da Bahia. Departamento de Educação Física. Salvador, BA. Brasil. Rev. Bras. Cineantropometria Desempenho Humano, 2011.

SILVA, A.S.; MANNARINO, I.C.; MOREIRA, A.B.; Risco nutricional em pacientes idosos hospitalizados como determinantes de desfecho clínico. Rev. Geriatria e Gerontologia, 2012.

TONINI, I.G.O.; LUZ, F.R.; MENON, P.D.; ZANELATTO, C.; MAZUR, C.E.; Percepção da saúde e estado nutricional de pacientes hospitalizados com doenças crônicas. Arquivos Brasileiros de Ciências e Saúde, 2018.

TRENTIN, AP, BERNARDI, JR, SIVIERO, J, Acompanhamento do estado nutricional e consumo alimentar de idosos durante o período de internação hospitalar. RBCEH, Passo Fundo, 2016.

WORLD HEALTH ORGANIZATION. Obesity: preventing and managing the global epidemic: Report of a WHO consultation on obesity. (WHO Technical Report Series n. 894). Geneva, Switzerland: WHO, 2000.

WORLD HEALTH ORGANIZATION. Physical Status: the use and interpretation of anthropometry. Geneva, Switzerland: WHO, 1995. (WHO Technical Report Series, n. 854.

ZANCHIM, M.C.; LIBERALI, R.; COUTINHO, V.; Estado Nutricional de Idosos hospitalizados em hospital geral de alta complexidade do Estado do Rio Grande do Sul. Rev. Bras Nutr Clin 2013; 28 (4): 292-9. 